\title{
La formación de investigadores con tecnologías digitales: una revisión sistemática de la literatura especializada
}

\author{
The Training of Researchers with Digital Technologies: a Systematic Review of \\ Specialised Literature \\ La formació d'investigadors amb tecnologies digitals: una revisió sistemàtica de la \\ literatura especialitzada
}

\author{
Rosalba Palacios-Díaz ${ }^{\circledR}$, Alexandro Escudero-Nahón* \\ Universidad Autónoma de Querétaro, Querétaro, México \\ *Autor para correspondencia: alexandro.escudero@uaq.mx (Alexandro Escudero-Nahón)
}

Recibido: 03/03/2020 | Aceptado: 13/05/2020 | Publicado: 24/06/2020

Cómo citar: Palacios-Díaz, $\mathrm{R}$, y Escudero-Nahón, A. (2020). La formación de investigadores con tecnologías digitales: una revisión sistemática de la literatura especializada. Research in Education and Learning Innovation Archives, 24,23-38. 10.7203/realia.24.16617

Copyright: El/La Autor/a. Open Access: Este es un artículo de acceso abierto distribuido bajo los términos de la licencia Creative Commons

Attribution-NoDerivatives 4.0 International licence (CC BY-ND 4.0)

Financiación: None informed
RESUMEN: La educación superior es uno de los tipos de educación más influidos por las tecnologías digitales. Esta situación, al tiempo que presenta varias ventajas, también impone muchos desafíos. La educación de tipo superior en México se conforma por tres niveles: técnico superior, licenciatura y posgrado. En los estudios de posgrado es donde se forman, propiamente, los investigadores en ciernes. No obstante, no existe abundante literatura especializada sobre los desafíos que impone las tecnologías digitales para formar a los investigadores en, relativamente, poco tiempo (de uno a cuatro años). Este estudio documental aplicó el método de investigación llamado Revisión Sistemática para identificar los hallazgos más relevantes que los especialistas han analizado sobre el uso de las tecnologías digitales por parte de los investigadores en formación. La Revisión Sistemática se realizó en cuatro fases: búsqueda documental en bases de datos científicos, descripción de los criterios de inclusión y exclusión de textos, análisis y categorización y, finalmente, discusión y propuesta. Los resultados más relevantes sugieren que los investigadores en formación lidian con varios problemas al momento de usar tecnología digital, entre los que destacan: la preparación incorrecta de los espacios de trabajo para la producción de conocimiento; la necesidad de generar articulaciones espontáneas entre las herramientas digitales disponibles, vigentes e interoperables; y la configuración adecuada de las redes de investigación, entre otros. Finalmente, se propone un proceso de uso de las tecnologías digitales que recorre, de forma itinerante, tres etapas durante la formación del investigador: preparación, uso y distribución.

PALABRAS CLAVE: tecnologías digitales; estudios de posgrado; formación de investigadores; tecnología educativa; revisión sistemática

ABSTRACT: Higher education is one of the types of education most influenced by digital technologies. This situation, while presenting several advantages, also imposes many challenges. Higher education in Mexico is made up of three levels: senior technician, graduate and postgraduate. In postgraduate studies it is where budding researchers are mean to be properly trained. However, there is no abundant literature on the challenges that digital technologies impose to train budding researchers in a relatively short period of time (from one to four years). This documentary study applied the research method called Systematic Review to identify the most relevant findings that specialists have analysed about the use of digital technologies by budding researchers in training. The Systematic Review was carried out in four phases: documentary search in scientific databases, 
description of the inclusion and exclusion criteria of texts, analysis and categorization and, finally, discussion and proposal. The most relevant results suggest that researchers in training deal with several problems when using digital technologies, such as: the inappropriate preparation of workspaces for the production of knowledge; the need to generate spontaneous articulations between the available, current and interoperable digital tools; and the inappropriate configuration of research networks, among others. Finally, we propose a process of use of the digital technologies that works with three stages during the researcher's training: preparation, use and distribution.

RESUM: L'educació superior és un dels tipus d'educació més influïts per les tecnologies digitals. Aquesta situació, al mateix temps que presenta diversos avantatges, també imposa molts desafiaments. L'educació de tipus superior a Mèxic es conforma per tres nivells: tècnic superior, llicenciatura i postgrau. En els estudis de postgrau és on es formen, pròpiament, els investigadors en germen. No obstant això, no hi ha abundant literatura especialitzada sobre els desafiaments que imposen les tecnologies digitals per a formar els investigadors en, relativament, poc temps (d'un a quatre anys). Aquest estudi documental va aplicar el mètode d'investigació anomenat revisió sistemàtica per identificar les troballes més rellevants que els especialistes han analitzat sobre l'ús de les tecnologies digitals pels investigadors en formació. La revisió sistemàtica es va dur a terme en quatre fases: cerca documental en bases de dades científiques, descripció dels criteris d'inclusió i exclusió de textos, anàlisis i categorització i, finalment, discussió i proposta. Els resultats més rellevants suggereixen que els investigadors en formació breguen amb diversos problemes en el moment d'usar tecnologia digital, entre els quals destaquen: la preparació incorrecta dels espais de treball per a la producció de coneixement; la necessitat de generar articulacions espontànies entre les eines digitals disponibles, vigents i interoperables; i la configuració adequada de les xarxes d'investigació, entre altres. Finalment, es proposa un procés d'ús de les tecnologies digitals que recorre, de manera itinerant, tres etapes durant la formació de l'investigador: preparació, ús i distribució.

PARAULES CLAU: tecnologies digitals; estudis de postgrau; formació d'investigadors; tecnologia educativa; revisió sistemàtica

\section{Notas de aplicación práctica}

\section{Qué se sabe sobre el tema}

- La educación superior es uno de los tipos de educación más influenciados por las tecnologías digitales.

- No hay abundante literatura sobre los desafíos que imponen las tecnologías digitales para capacitar a los investigadores en ciernes.

- Los pocos estudios que abordan el desafío de capacitar a los investigadores con tecnologías digitales han identificado que los investigadores en ciernes realizan prácticas de aprendizaje predominantemente individuales.

\section{Qué aporta este trabajo}

- Un conocimiento de vanguardia del aprendizaje digital en la formación de investigadores en ciernes.

- Una propuesta de tres categorías de análisis sobre aprendizaje digital en la formación de investigadores en ciernes.

- Una justificación de la necesidad de un modelo de aprendizaje digital en la formación de investigadores en ciernes.

\section{Implicaciones para la práctica y / o política}

- Una atención especial a las prácticas de aprendizaje con tecnologías digitales de investigadores en ciernes.

- La necesidad de construir un modelo de aprendizaje para los investigadores en ciernes que utilizan tecnologías digitales.

\section{INTRODUCCIÓN}

La educación de tipo superior en México se conforma por tres niveles: técnico superior, licenciatura y posgrado. El técnico superior se orienta a la formación de profesionales 
capacitados para el trabajo en un área específica. Las licenciaturas forman profesionistas en diversas áreas del conocimiento con programas de estudio de cuatro años o más; se imparten en instituciones universitarias, tecnológicas y de formación de maestros y es de carácter terminal. El posgrado incluye los estudios de especialidad, maestría y doctorado. Está orientado a la formación de investigadores y profesionistas con alto grado de especialización. El posgrado tiene como requisito obligatorio de ingreso la licenciatura y se acredita con el título de especialidad, maestría o doctorado (DOF, 2018).

El nivel donde se forman, propiamente, los investigadores en México, es en el posgrado. Dependiendo del plan curricular de la especialidad, la maestría o el doctorado, dichos investigadores en ciernes tendrán entre uno y cuatro años de formación al respecto.

La educación superior, actualmente, es uno de los tipos de educación más influidos por las tecnologías digitales. Esta situación, al tiempo que presenta varias ventajas, también impone varios desafíos. Entre los desafíos más importantes se encuentran: la consolidación de una cultura de la innovación que comprenda el uso de las tecnologías digitales; la evaluación integral del aprendizaje con el uso de las tecnologías digitales; y la reorganización de las instituciones para incorporar adecuadamente las tecnologías digitales (Alexander et al., 2019).

Dichos desafíos han sido descritos pormenorizadamente por los especialistas en educación superior y tecnología digital y, también, han sido planteados algunos objetivos a mediano y largo plazo en la agenda educativa contemporánea. No obstante, no existe abundante literatura especializada sobre los desafíos que impone las tecnologías digitales para formar a los investigadores en, relativamente, poco tiempo (de uno a cuatro años).

Desde el punto de vista teórico, ha sido la Teoría Postdigital quien ha elevado una propuesta de estudio al respecto. El término postdigital, propiamente dicho, apareció mencionado por primera vez en el libro La membrana postdigital: imaginación, tecnología y deseo (Pepperell y Punt, 2000). En ese documento, la preocupación principal era que varios procesos biológicos, fisiológicos y orgánicos estaban siendo codificados de manera binaria, como son los procesos digitales, y se estaban difuminando los límites entre lo biológico y lo sintético; entre lo virtual y lo real; entre lo orgánico y lo inorgánico; entre lo digital y lo análogo; entre lo natural y lo artificial; en fin, entre lo humano y lo no humano.

Esta condición postdigital, entendida como la incorporación amplia, profunda y transversal de las tecnologías digitales en la vida cotidiana fue el punto de partida para fundar la Teoría Postdigital y para justificar, en consecuencia, el desarrollo del concepto Educación Postdigital. La Teoría Postdigital es una propuesta muy reciente. De acuerdo con sus fundadores (Jandrić, 2019, p. 1), está inspirada en una de las ideas más provocadoras de Nicholas Negroponte, reconocido arquitecto e informático estadounidense de origen griego, fundador y director del Media Lab del Instituto Tecnológico de Massachusetts (MIT, por sus siglas en inglés). Hace veinticuatro años, Negroponte publicó un libro referencial titulado Being digital (Negroponte, 1995), donde hizo un análisis de la evolución de varias tecnologías e intentó predecir cómo influirían en la vida cotidiana. Su deducción fue que varias funciones vitales de la humanidad se digitalizarían, es decir, que se trasladarían del mundo orgánico, compuesto por átomos, hacia plataformas digitales, compuestas por bits.

Tres años después el mismo autor publicó el artículo "Más allá de lo digital" en la revista de divulgación científica Wired, de la cual es socio fundador (Negroponte, 1998). En ese artículo aseguró, categóricamente, que la revolución digital había terminado. $\mathrm{Su}$ argumento fue que la forma literal del mundo digital, es decir, la tecnología, ya se había dado por sentada en varios ámbitos sociales, como la economía, la salud, el 
arte, la cultura y, por supuesto, la educación. De tal manera que las próximas innovaciones tendrían como punto de partida, invariablemente, a las tecnologías digitales. Esta amplia, profunda y transversal incorporación de las tecnologías digitales en la vida cotidiana de las personas plantearía una situación inédita en la historia de la humanidad. En palabras del propio autor: "Al igual que el aire y el agua potable, ser digital solo se notará por su ausencia, no por su presencia (Negroponte, 1998, p. 1).

$\mathrm{El}$ argumento central, como puede apreciarse, es que ya no es pertinente hacer una distinción entre la educación digital y la educación no digital porque las plataformas y los entornos de aprendizaje han superado esa dicotomía simplista. Por eso, se propone la convergencia de varias disciplinas del conocimiento como la pedagogía crítica, la educación digital, el aprendizaje en red, el posthumanismo, la filosofía, la política, las artes, entre otras, en torno a la condición postdigital de la educación (Jandrić et al., 2018).

Hasta el momento, no se ha definido con claridad qué es la Educación Postdigital. A lo más, se ha dicho que esta teoría intenta describir cómo afectan las actuales relaciones humanas y las tecnologías digitales a los procesos educativos; se ha mencionado, también, que esta teoría interpela la participación de varias disciplinas de estudio; se ha descrito, además, que esta teoría considera como una situación ambigua el hecho de que, actualmente, conviven las tecnologías analógicas y las digitales, el pensamiento posthumanista y el pensamiento postmoderno, la crítica al capitalismo del conocimiento y al capitalismo bio-informacional; en fin, solamente se ha dicho que la educación experimenta un "eclecticismo rampante" (Jandrić et al., 2018, p. 894).

En realidad, la Educación Postdigital solamente ha generado un debate académico de cierto interés sobre la educación, pero no ha sido capaz de crear categorías de análisis al respecto. Los propios fundadores del concepto han mencionado que quizás preferirían un concepto nuevo, pero, por lo pronto, consideran que el concepto Educación Postdigital es adecuado a la condición postdigital (Jandrić et al., 2018, p. 896). Por lo anterior, es perentorio realizar análisis conceptuales críticos sobre la Educación Postdigital con el objetivo de valorar su pertinencia para analizar la formación de los investigadores en ciernes con tecnologías digitales.

Hasta el momento, no se ha definido con claridad qué es la Educación Postdigital. A lo más, se ha dicho que esta teoría intenta describir cómo afectan las actuales relaciones humanas y las tecnologías digitales a los procesos educativos; se ha mencionado, también, que esta teoría interpela la participación de varias disciplinas de estudio; se ha descrito, además, que esta teoría considera como una situación ambigua el hecho de que, actualmente, conviven las tecnologías analógicas y las digitales, el pensamiento posthumanista y el pensamiento postmoderno, la crítica al capitalismo del conocimiento y al capitalismo bio-informacional; en fin, solamente se ha dicho que la educación experimenta un "eclecticismo rampante" (Jandrić et al., 2018, p. 894). Por eso, los procesos educativos contemporáneos deberían ser estudiados con nuevas categorías de análisis, tomando en cuenta que en el futuro próximo se realizarán, a la vez, con lo digital y lo analógico; lo virtual y lo real; lo biológico y lo sintético; en suma, con participación de agencias humanas y no humanas (Ford, 2019; Peters y Jandrić, 2019).

Por lo anterior, es perentorio realizar revisiones sistemáticas de la literatura especializada con el objetivo de identificar cómo se forman los investigadores con el uso de tecnologías digitales y si el concepto Educación Postdigital es el más adecuado al respecto.

Los pocos estudios que abordan el desafío de formar investigadores con tecnología digital han identificado que, en el nivel posgrado, los investigadores realizan prácticas de aprendizaje predominantemente individuales, aun cuando las tecnologías digitales permite el trabajo colaborativo (Arras-Vota, Gutiérrez-Díez, y Bordas-Beltrán, 2010). 
Además, la exigencia que imponen las instituciones de educación superior a los investigadores en ciernes para realizar investigaciones de frontera se resuelve, comúnmente, sin seguir un plan al respecto, sino con prácticas personales, no institucionales, espontáneas, intuitivas, exploratorias de las opciones que ofrece las tecnologías digitales. Por ejemplo, los investigadores en ciernes aprenden a usar las bases de datos científicas, los gestores de referencia, las aplicaciones que apoyan la investigación, a través de video tutoriales y con ayuda de sus pares, principalmente.

Sumado a este alejamiento del aprendizaje formal e institucional, generalmente, la investigación formativa que realizan los estudiantes de posgrado debe alternarse con otras actividades académicas, como la docencia, la gestión, la divulgación y la difusión de la ciencia. Es decir, el investigador en ciernes pone en juego todas sus destrezas para administrar los escasos recursos de tiempo, dinero y equipo humano con los que cuenta, para cumplir con las exigencias institucionales sobre la investigación: obtener financiamiento para investigar, presentar resultados relevantes, difundirlos y divulgarlos entre la comunidad científica, y consolidar una línea de investigación original (Casimiro, Casimiro, y Casimiro, 2017). Así que la mayoría de las actividades que realizan los investigadores en ciernes son el resultado de decisiones tomadas con base en su capacidad de gestión y su pericia para adquirir conocimiento sobre el uso de las tecnologías digitales en el ámbito investigativo por vías no formales.

Aunque existen algunas iniciativas para incorporar las tecnologías digitales de manera sistematizada en la educación superior, varios estudios muestran que generalmente provoca varias reticencias entre los docentes y su uso termina siendo una adecuación de la didáctica tradicional. Varios estudios realizados sobre la autopercepción que tiene la comunidad universitaria respecto a la incorporación de las tecnologías digitales en los procesos educativos muestran que se valora, principalmente, el carácter tecnológico e instrumental. Sin embargo, las tecnologías digitales no se valoran, en sí mismas, como un elemento transformador de la dimensión pedagógica (CaberoAlmenara, 2013).

Lo anterior sugiere la siguiente pregunta de investigación: ¿Cómo utilizan las tecnologías digitales los investigadores en formación?

\section{MÉTODO}

El objetivo de esta investigación documental fue identificar los hallazgos más relevantes que los especialistas han analizado sobre el uso de las tecnologías digitales por parte de los investigadores en formación. La selección de este grupo poblacional es relevante por dos motivos: primero, porque se ha documentado previamente en investigaciones empíricas que no existe abundante investigación científica sobre el postgrado y el uso de tecnologías digitales, al tiempo que se ha advertido la necesidad de que se realice más investigación acerca de la gestión y calidad de programas educativos, la virtualización de la educación, niveles, modelos y modalidades de educación mediados con tecnología, y estudios referentes a política educativa y cambio social (Olivares-Carmona, Angulo-Armenta, Torres-Gastelú, y Madrid-García, 2016); segundo, de acuerdo con las autoridades responsables de desarrollar la investigación y financiar la formación de los investigadores en México, es decir el Consejo Nacional de Ciencia y Tecnología, la misión de desarrollar y fortalecer las capacidades científicas, tecnológicas y de innovación nacionales se logra a través del apoyo a proyectos directamente vinculados con la investigación en ciencias y humanidades, con el desarrollo tecnológico, con la innovación, con la formación especializada y altamente calificada de la comunidad académica y de investigadores (Conacyt, 2020).

Para identificar los hallazgos más relevantes que los especialistas han analizado sobre el uso de las tecnologías digitales por parte de los investigadores en formación, se realizó una Revisión Sistemática de la literatura especializada en bases de datos 
científicos (Gisbert y Bonfill, 2004; Sánchez-Meca, 2010). La Revisión Sistemática se realizó en cuatro etapas:

1. Búsqueda documental sobre el uso de las tecnologías digitales por parte de los investigadores en formación. Se realizó la búsqueda el 20 de enero de 2020 en las siguientes bases de datos: Science Direct, Redalyc, Conricyt y DOAJ. Estas bases de datos científicos fueron seleccionadas con los siguientes criterios: era necesario contar con literatura especializada de, por lo menos, una base de datos de las dos empresas que detentan la indización de más revistas mundialmente (Science Direct); era necesario contar con literatura especializada de, por lo menos, una base de datos de Latinoamérica, para obtener literatura localizada en este territorio, que no necesariamente aparece en bases de datos internacionales (Redalyc); era necesario contar con literatura especializada de, por lo menos, una base de datos mexicana, para obtener literatura localizada en este territorio que no necesariamente aparece en bases de datos latinoamericanas (Conricyt); era necesario contar con literatura especializada de, por lo menos, una base de datos de acceso abierto (DOAJ).

2. Descripción de los criterios de selección de textos. En este estudio se ha definido el uso de las tecnologías digitales en el trabajo de investigación en cuatro ámbitos: investigador que mejora su propio aprendizaje; investigador con propósito de mejorar el aprendizaje de otras personas; investigador que mejora su propio aprendizaje sin intencionalidad; e investigador que mejora el aprendizaje de otras personas sin intencionalidad (Figura 1).

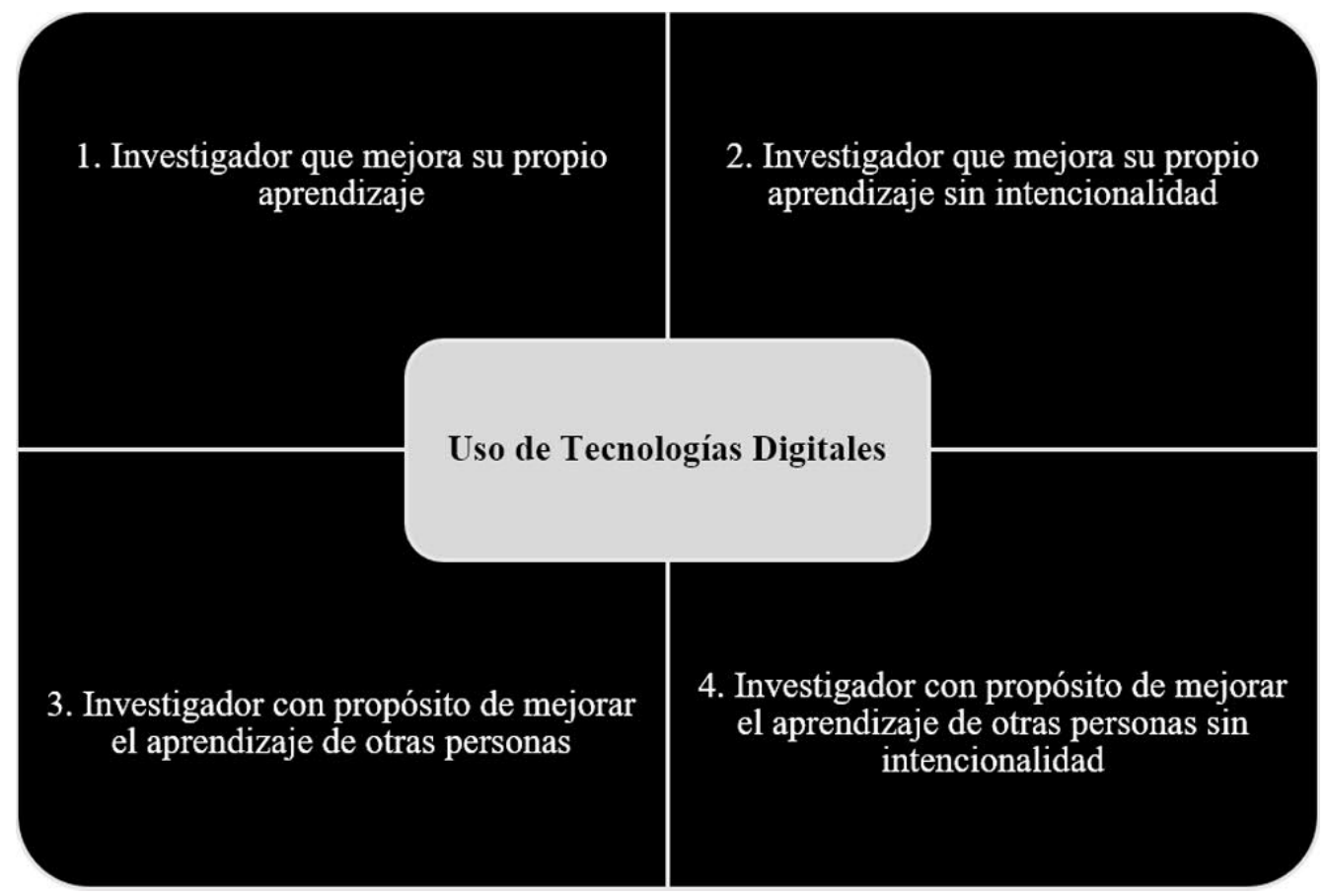

Figura 1. Ámbitos considerados enla Revisión Sistemática de acuerdo con el uso de lastecnologías digitales en el trabajo de investigación. Fuente: elaboración propia.

Esta investigación concentró la revisión de textos en el primer ámbito, es decir, el del investigador que mejora su propio aprendizaje. Esto supuso seleccionar y analizar aquellos estudios que abordaron la intervención de las tecnologías digitales en la formación del investigador. En principio, se realizó la búsqueda en inglés, pero las bases de datos arrojaron muy pocos textos y ninguno de ellos era pertinente a este estudio. Sabedores de que un artículo científico debe revisar la literatura científica 
internacional para poder expresar conclusiones, pero ante la imposibilidad de obtener literatura relevante en inglés, se optó por hacer la búsqueda solamente en español. Para este propósito, la búsqueda de términos en las bases de datos se segmentó de la siguiente manera: ("investigador") AND ("aprendizaje digital"). Se obtuvo el siguiente número de documentos: Science Direct, 46; Redalyc, 50; DOAJ, 16; Conricyt, 4. Se aceptaron estudios en español publicados desde 2015 hasta 2019, por ser un tema de investigación de pertinencia regional. La selección incluyó investigación empírica y documental. A continuación, se realizó la eliminación de documentos duplicados. Después, se seleccionaron los documentos congruentes con el tema investigado, a partir de la revisión del título, resumen y palabras clave. Se obtuvieron 20 documentos para la posterior etapa de análisis.

3. Análisis y categorización. El periodo de análisis se realizó desde el 21 hasta el 28 de enero de 2020. Las preguntas que guiaron el análisis cuantitativo se presentan en la Tabla 1. El objetivo del análisis cuantitativo fue conocer cuántas publicaciones por año se han realizado sobre el uso de las tecnologías digitales por parte de los investigadores en formación, qué áreas de conocimiento abordaron el tema y en qué países se ha publicado más al respecto.

Tabla 1. Preguntas de investigación del análisis cuantitativo. Fuente: elaboración propia

\begin{tabular}{ll}
\hline Temas de análisis & Preguntas de investigación \\
\hline $\begin{array}{ll}\text { Número de publicaciones } \\
\text { por año }\end{array}$ & $\begin{array}{l}\text { ¿Cuántos documentos por año se han publicado sobre el uso de las } \\
\text { tecnologías digitales por parte de los investigadores en formación? }\end{array}$ \\
$\begin{array}{l}\text { Áreas de conocimiento } \\
\text { que han publicado }\end{array}$ & ¿Qué áreas del conocimiento han publicado estudios sobre sobre el uso de \\
estudios & las tecnologías digitales por parte de los investigadores en formación? \\
Países que han publicado & $\begin{array}{l}\text { ¿Qué países han publicado sobre sobre el uso de las tecnologías digitales } \\
\text { por parte de los investigadores en formación? }\end{array}$ \\
\hline
\end{tabular}

Asimismo, se realizó un análisis cualitativo para describir los problemas más relevantes sobre el uso de las tecnologías digitales por parte de los investigadores en formación, con qué métodos de investigación se ha analizado este tema y qué resultados relevantes se han obtenido. Las preguntas de investigación que condujeron el análisis cualitativo se presentan en la Tabla 2.

4. Discusión. La discusión se presenta, propiamente, como parte de las Conclusiones de este texto.

\section{ANÁLISIS Y RESULTADOS}

\subsection{Resultados}

El análisis cuantitativo no fue profundo, solo tuvo como objetivo identificar:

- ¿Cuántos documentos por año se han publicado sobre el uso de las tecnologías digitales por parte de los investigadores en formación?

- ¿Qué áreas del conocimiento han publicado estudios sobre el uso de las tecnologías digitales por parte de los investigadores en formación?

- ¿Qué países han publicado sobre el uso de las tecnologías digitales por parte de los investigadores en formación?

La Revisión Sistemática mostró un mayor número de publicaciones en el 2018. No se percibe una tendencia creciente del tema. La producción de textos dedicados al uso de las tecnologías digitales en la investigación es reducida. Sin embargo, se observa una consistencia en el número de estudios por año (Figura 2). 
Tabla 2. Preguntas de investigación del análisis cualitativo. Fuente: elaboración propia

\begin{tabular}{|c|c|}
\hline Temas de análisis & Preguntas de investigación \\
\hline Problemas relevantes. & $\begin{array}{l}\text { ¿Qué problemas consideran las investigaciones } \\
\text { especializadas como relevantes sobre el uso de } \\
\text { las tecnologías digitales por parte de los } \\
\text { investigadores en formación? }\end{array}$ \\
\hline Métodos de investigación. & $\begin{array}{l}\text { ¿Qué métodos de investigación se han utilizado } \\
\text { para analizar el uso de las tecnologías digitales } \\
\text { por parte de los investigadores en formación? }\end{array}$ \\
\hline Resultados relevantes. & $\begin{array}{l}\text { ¿Qué resultados relevantes se han obtenido sobre } \\
\text { el uso de las tecnologías digitales por parte de los } \\
\text { investigadores en formación? }\end{array}$ \\
\hline
\end{tabular}

Los países que han publicado sobre el aprendizaje digital en la investigación son Argentina ( 2 artículos), Chile (1 artículo), Colombia (1 artículo), Cuba (1 artículo), Ecuador (1 artículo), España (4 artículos), España-Alemania (1 artículo), Estados UnidosEspaña (1 artículo), México (5 artículos), México-España (1 artículo), Perú (1 artículo) y Venezuela (1 artículo). El mayor porcentaje de publicación lo obtuvo México con un $25 \%$, seguido por España con un 20\%, mientras que diversos países hispanohablantes publicaron una mínima cantidad de documentos (Figura 3).

Se utilizó como referencia el Manual del Sistema de Clasificación de Revistas Mexicanas de Ciencia y Tecnología para establecer las áreas del conocimiento que han analizado los problemas relacionados con el aprendizaje digital en la investigación (Conacyt, 2018). Estas áreas del conocimiento son: Física, Matemáticas y Ciencias de la Tierra; Biología y Química; Medicina y Ciencias de la Salud; Humanidades y Ciencias de la Conducta; Ciencias Sociales; Biotecnología y Ciencias Agropecuarias; Ingenierías; Multidisciplinarias (Figura 4). Se observó un mayor número de publicaciones del área de Humanidades y Ciencias de la Conducta; Medicina y Ciencias de la Salud; y, Multidisciplinarias. Mientras que el resto de las áreas de conocimiento no reportaron estudios publicados.

En este texto se mencionó que fueron tres las preguntas que guiaron el análisis cualitativo. La primera pregunta fue ¿qué problemas consideran las investigaciones

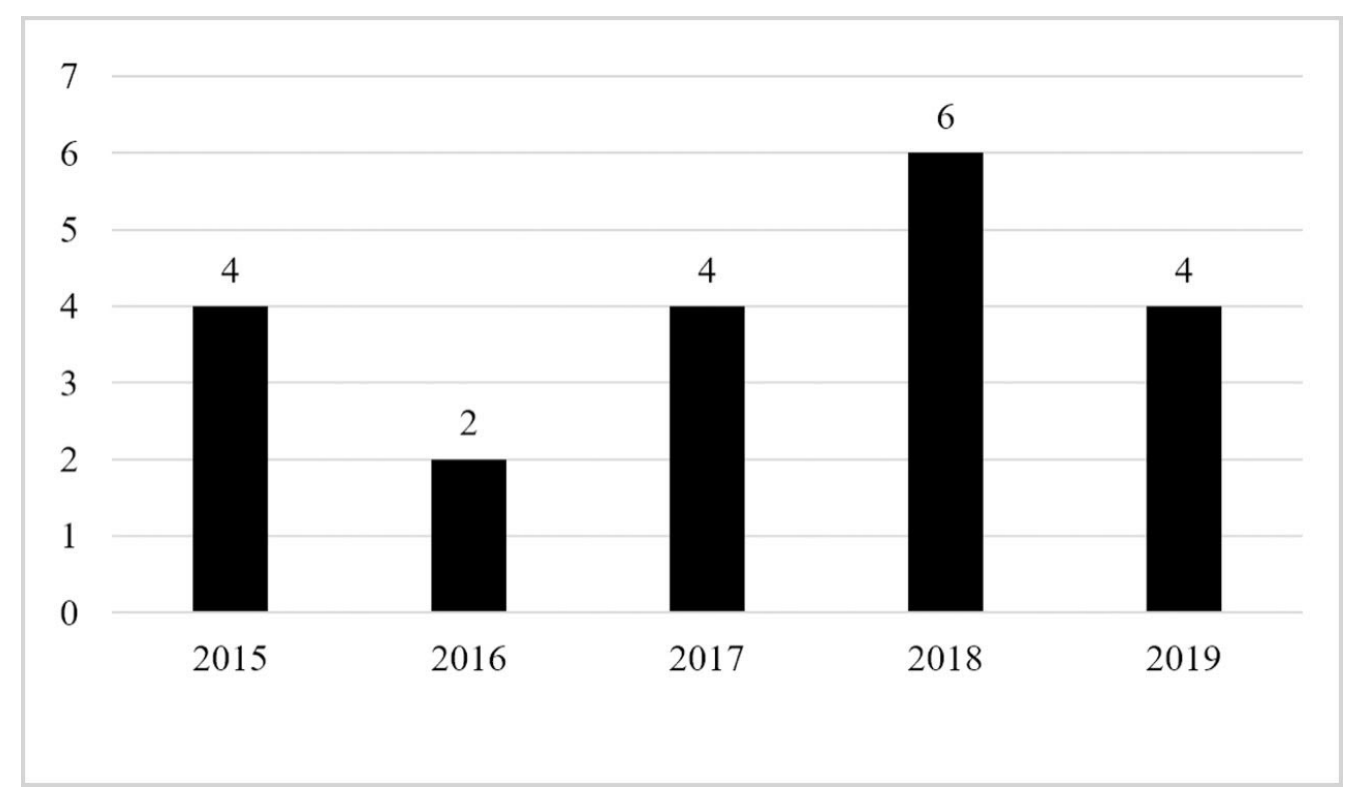

Figura 2. Número de publicaciones por año. Fuente: elaboración propia. 


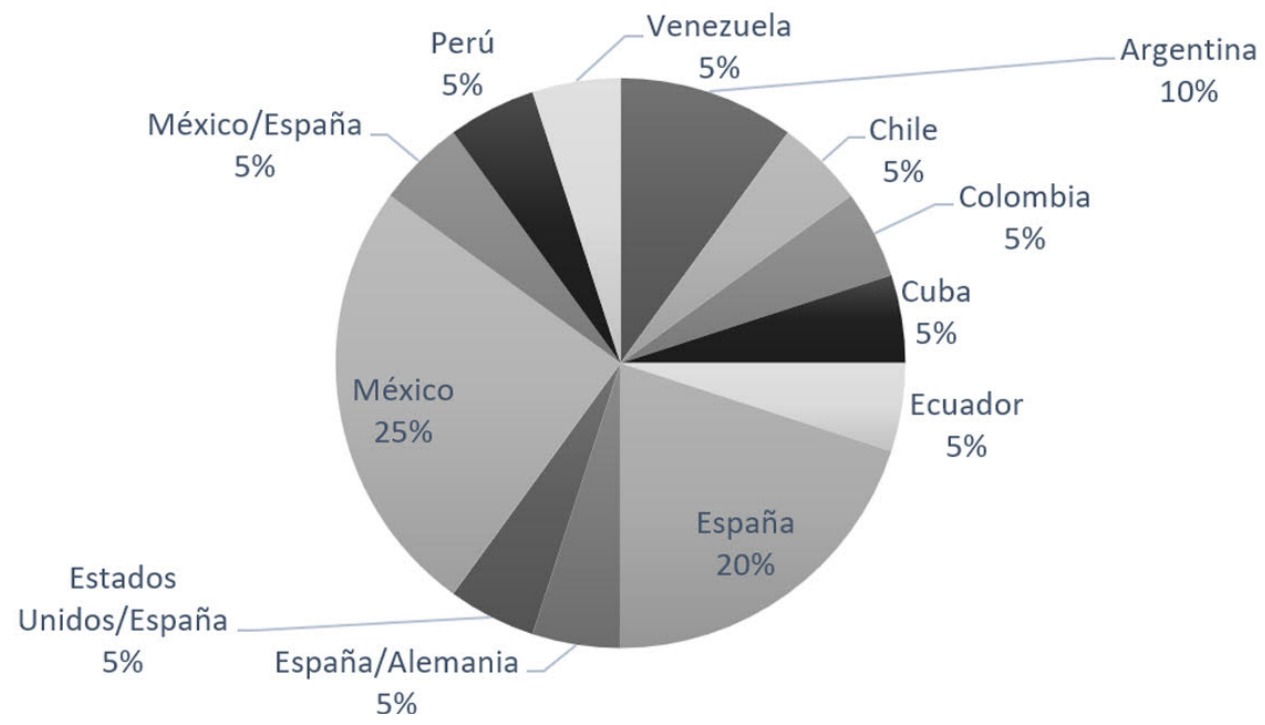

Figura 3. Número de publicaciones por país. Fuente: elaboración propia.

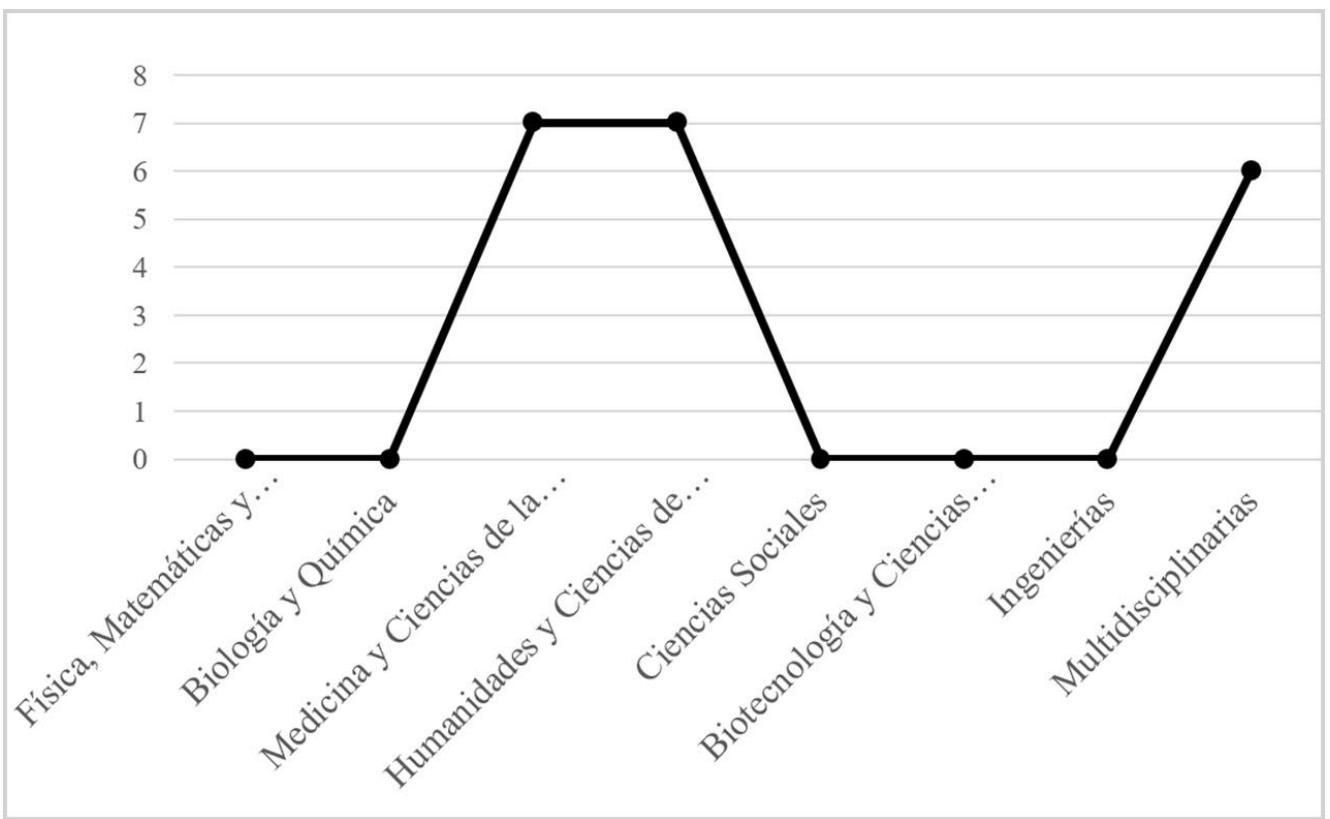

Figura 4. Áreas del conocimiento que han abordado el tema del aprendizaje digital en la educación. Fuente: elaboración propia.

especializadas como relevantes sobre el uso de las tecnologías digitales por parte de los investigadores en formación?

Lo anterior permitió obtener cuatro categorías generales:

1. Estructura y organización

2. Herramientas de aprendizaje digital

3. Escenarios de aprendizaje

4. Redes de colaboración

A continuación, el proceso de análisis cualitativo a detalle. 
En la categoría 1) Estructura y organización, se encontraron los problemas que ubican a las tecnologías digitales en un estado preparatorio, es decir, aquellos problemas que abordan la configuración de los entornos digitales en el ámbito de la investigación. En este sentido, se detectó la urgencia de proveer al investigador de recursos documentales en su versión digital con disponibilidad y servicio competitivos (MuirraguiIrrazábal, 2019). También, la necesidad de cambio cultural y tecnológico en el rol del investigador (Sancho-Gil, Ornellas, y Arrazola-Carballo, 2018). Se observó además, la importancia de la identificación oportuna de la configuración de Universidad Digital, esto es, reconocer su práctica, su enfoque, su tendencia y su trabajo en red (SalinasIbáñez y Irene-Marin, 2018) y la necesidad de tipificar a los grupos de estudio en torno a sus líneas de investigación y su comunicación tecnológica (Sime-Poma, 2017). Otro problema fue la falta de infraestructura y equipamiento tecnológico congruente con el campo disciplinar que lo demande (Becerra-Romero, 2017). Finalmente, se encontró la exigencia de una transformación orientada hacia la epistemología de la investigación en la sociedad del conocimiento (Carrasco, Baldivieso, y Di-Lorenzo, 2016).

En la categoría 2) Herramientas del aprendizaje, se situaron problemáticas cuyas soluciones residían en el diseño y uso de objetos digitales que fortalecieran la adquisición de competencias del investigador, tales como: la búsqueda de información, la comprensión de conceptos o el trabajo colaborativo. En este marco se registró la necesidad de revistas digitales científicas con disponibilidad, frecuencia de publicación, calidad y relevancia (Navarrete y Pérez, 2019; Rodríguez-Bravo, Alvite-Díez, y OleaMerino, 2015). También, se asentaron las características de los objetos de aprendizaje para la investigación, como la vigencia, la reutilización, la interoperabilidad y la disponibilidad (Degkwitz, 2017; Hernández, Rodríguez, Andrés-Pérez, y Augusto-Reyes, 2017; Marzal, Calzada-Prado, y Ruvalcaba-Burgoa, 2015; Pisté-Beltrán y and, 2019). Para concluir, se identificó la necesidad de la gestión de datos para la investigación a través de técnicas de innovación (Fernández-Altunaa et al., 2016; Núñez-Reiz, de-la Hoz, y Sánchez-García, 2019).

En la categoría 3) Escenarios de aprendizaje, se encontraron las problemáticas con respecto a la configuración de los entornos de aprendizaje digitales. Por ejemplo, la educación digital sin modelos pedagógicos apropiados que resulta en una preparación ineficiente de los investigadores (López-Ledesma, Álvarez, y Bassa, 2018; RubioHurtado, Vilà-Baños, y Berlanga-Silvente, 2015). También se identificó la satisfacción parcial que ofrecen los programas de educación virtual, pues carecen de esquemas que incorporen la actualización y el trabajo en grupos de investigación (Arras-Vota et al., 2010). Se incluye además, la falta de programas dedicados al estímulo de la producción científica (Chávez-Caraza, de Ita, Lozano-Ramírez, Vargas-Duarte, y Lozano-Lee, 2015).

En la categoría 4) Redes de colaboración, se encontraron las dificultades para generar grupos y redes de investigación que, con intervención tecnológica, cumplan con el propósito de la producción científica. En tal sentido, se analizó la falta de herramientas de uso compartido en el marco de la web 2.0 (Santana, 2018). Otro problema fue la carencia del uso efectivo de las redes sociales con relación a la difusión y vinculación de la investigación (Gómez-Rivas et al., 2019; Guerrero-Martínez y Reyes, 2018). Es importante señalar que las redes de colaboración estuvieron presentes durante la confección de varios estudios pertenecientes a las tres categorías antes mencionadas, aunque sin un papel protagónico en el análisis.

La segunda pregunta que condujo el análisis cualitativo fue ¿qué métodos de investigación se han utilizado para analizar el uso de las tecnologías digitales por parte de los investigadores en formación? Se han utilizado diversos métodos para analizar el aprendizaje en la investigación (Figura 5).

Se destacaron los métodos cualitativos, cuantitativos y descriptivos con diversos instrumentos de análisis de datos. También, se emplearon el estudio de caso y la 


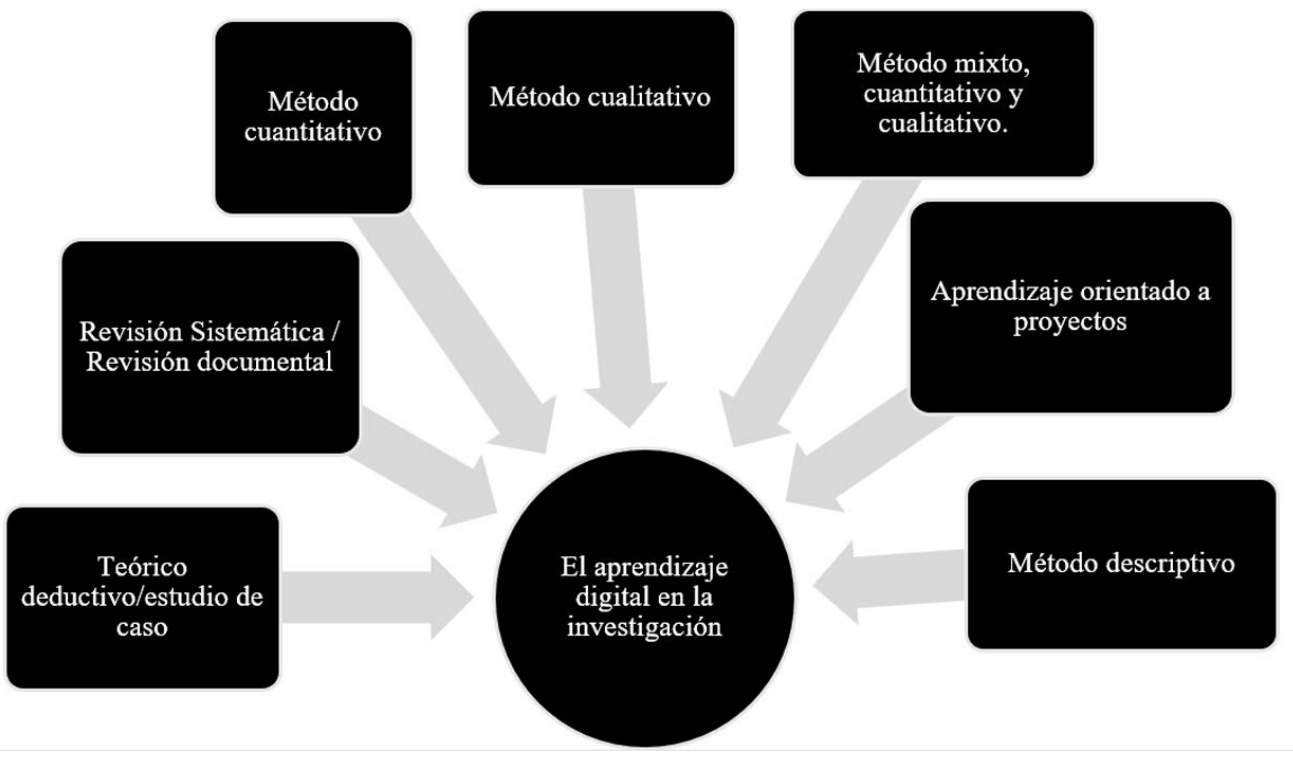

Figura 5. Métodos de investigación para analizar el aprendizaje en la investigación.

Fuente: elaboración propia.

revisión sistemática. Por último, se observó el uso de métodos aplicados al ámbito educativo como el aprendizaje orientado a proyectos (Figura 5).

La tercera pregunta que condujo el análisis cualitativo fue ¿qué resultados relevantes se han obtenido sobre el uso de las tecnologías digitales por parte de los investigadores en formación? Para responder a esta cuestión, se ha ordenado el escenario del fenómeno de estudio en una propuesta que recorre las etapas de cómo utilizan las tecnologías digitales los investigadores en formación (Figura 6).

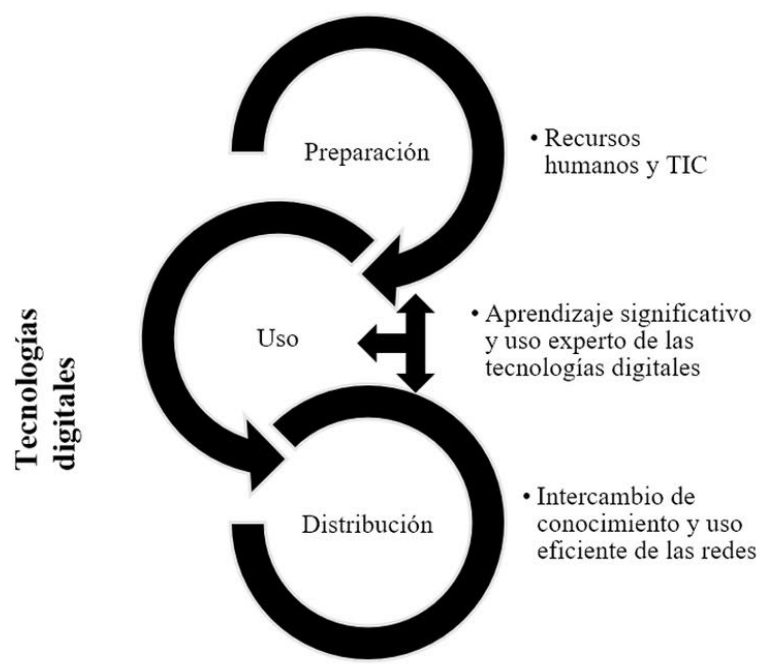

Figura 6. Propuesta del proceso en el uso de las tecnologías digitales por los investigadores en formación. Fuente: elaboración propia.

A partir de este proceso, se proponen tres etapas de tránsito del aprendizaje digital en la investigación. Dichas fases son descritas a continuación:

- Etapa de preparación. En esta etapa se prepara al recurso tecnológico, humano e institucional para el trabajo del investigador con las tecnologías digitales. Así pues, se valoran las tecnologías, la infraestructura, los entornos de aprendizaje, los 
modelos disciplinares, los enfoques de investigación y las redes de conocimiento que utilizará el investigador durante su experiencia con el uso de las tecnologías digitales.

- Etapa de uso. En esta etapa ocurre el uso de herramientas y entornos virtuales pertinentes al desarrollo del perfil del investigador. Algunos ejemplos de los elementos que se accionan en esta etapa son: los programas de investigación en línea, los objetos virtuales de aprendizaje, las métricas digitales, el Big Data y las revistas científicas digitales, por mencionar algunos.

- Etapa de distribución. En esta etapa se almacena, busca, distribuye y se selecciona el conocimiento digital a través del uso de redes y grupos de investigación en el marco de las nuevas tecnologías de información y comunicación (TIC).

Como se puede observar en el diagrama, estas etapas pueden entablar acciones de retroalimentación gracias a las propiedades de ubicuidad en la red digital.

\section{DISCUSIÓN Y CONCLUSIONES}

El objetivo de este estudio fue responder a la pregunta de investigación ¿cómo utilizan las tecnologías digitales los investigadores en formación? Una primera reflexión, que ha sido fundada durante la realización de este estudio, es la posición de la comunidad científica frente a las tecnologías digitales.

Dicha comunidad ha recibido de forma positiva los enfoques y retos que surgen de la intervención de las tecnologías digitales en la producción del conocimiento. Sin embargo, los roles educativos que producen las herramientas y entornos de aprendizaje que forman al investigador, no se actualizan con la misma velocidad que los avances en las TIC (Carrasco et al., 2016). Esta condición hace necesario el trabajo interdisciplinario para identificar y resolver los problemas de la falta de paridad entre los roles pedagógicos y los avances en TIC.

En este sentido, se vuelve apremiante la formación pertinente y oportuna de los investigadores de cara a la sociedad y economía del conocimiento. En este marco, la masa crítica de investigadores es la que reconoce la transición de una economía basada en la explotación de los recursos naturales y empleos operativos, hacia una fundada en los hallazgos de la ciencia y la tecnología (Powell y Snellman, 2004). Esta circunstancia, en conjunto con la poca producción empírica y documental sobre cómo utilizan las tecnologías digitales los investigadores en formación, conceden el carácter de relevante a la exploración de esta temática.

Se observa, también, que hay áreas del conocimiento como la Física, Matemáticas y Ciencias de la Tierra; Biología y Química; Biotecnología y Ciencias Agropecuarias; y, las Ingenierías; que no han mostrado interés en documentar la forma en la que el investigador en formación utiliza la tecnología. En este aspecto, dichas áreas de conocimiento emplean las tecnologías digitales por su carácter instrumental y no han explorado su dimensión pedagógica. No obstante, sería interesante revisar qué ha sucedido con los textos científicos acerca de la formación digital del investigador producidos por las áreas del conocimiento previamente citadas.

Por otro lado, algunos grupos de investigación de habla hispana, que trabajan con las tecnologías digitales, se encuentran en una fase de autoconocimiento y organización. Por ejemplo, existen disciplinas que realizan cursos piloto virtuales para generar interés en la investigación y obtener perfiles idóneos de candidatos (Chávez-Caraza et al., 2015). Casos como este exponen la relevancia de reconocer la categoría a la que pertenece un grupo de investigación debido a que, una vez tipificado, un cuerpo de investigadores puede emprender la elaboración de estrategias que ayuden a mejorar sus prácticas y a planear las estrategias de instrucción de los programas académicos. 
Por estas razones, reconocer y organizar al grupo de investigación dentro de una tipificación, contribuye a forjar la identidad del organismo dentro de la red de conocimiento digital (Sime-Poma, 2017). Así que los investigadores en formación enfrentan el reto de aprovechar y aprender de las tecnologías digitales que tienen a su disposición. No siempre serán espacios idóneos. En ocasiones, los entornos no se habrán diseñado de acuerdo con los objetivos e identidad de los cuerpos de investigación. Pero una vez que el investigador determine su enfoque disciplinar, sus líneas de estudio y sus redes de intercambio, será capaz de seleccionar las tecnologías digitales apropiada para las tareas que contribuirán a su formación y producción. Se habrá superado, entonces, la etapa de preparación y comenzará la fase de uso, donde se hará una interacción intensa con las herramientas y espacios digitales.

En la etapa de uso se recorren varios procesos de investigación que han sido paulatinamente digitalizados, tales como: la búsqueda de fuentes información científica, la publicación del conocimiento y la gestión de datos, por mencionar algunos. Sin embargo, la aparición de problemas en el proceso de digitalización, como la falta de modelos pedagógicos para los aprendizajes virtuales o las características limitadas de las herramientas digitales indican la ausencia o la escasez del trabajo multidisciplinario, al diseñar productos educativos para los investigadores. Estas carencias pueden ser saldadas con la incorporación de expertos en las disciplinas de pedagogía o el diseño web, entre otros. Encontrar cómo se aprende mejor, además de entender los vínculos entre pedagogía y tecnología, puede potencializar el proceso formativo del investigador (Gros, 2016). Esta etapa de uso, así como la etapa de la preparación, recurren durante la ejecución de sus tareas a la fase de la distribución.

Dicha fase manifiesta también otra circunstancia, pues en la actualidad las redes de información y comunicación están presentes de manera constante. La acción del investigador en ciernes se da en un espacio conectado, pero los problemas que ocurren en esta etapa pueden asociarse a la complejidad de la red. El investigador es un agente que entra en diálogo con múltiples instancias dentro de la red y de forma itinerante, tal como lo sugiere la Teoría del Actor Red (Vaccari, 2008). Estos espacios pueden ser formales como las revistas digitales, o informales como las redes sociales. En este marco y en concordancia con la teoría de la conectividad se sugiere que la transferencia del conocimiento sucede en un entorno donde la información es recuperada dentro y fuera del individuo (Gros y Noguera-Fructuoso, 2013). Es decir, el aprendizaje es una experiencia de inmersión, ubicua y atemporal. Se accede entonces, a recursos situados en diversas modalidades, por ejemplo, vídeos o cursos en línea; y temporalidades, es decir, en tiempo real o diferido. Bajo estas condiciones el trabajo colaborativo, la alfabetización digital, así como la interoperabilidad entre modalidades son ejes de la formación en los individuos conectados. Sin embargo, estas instancias no garantizan que la información digital recibida por un investigador transite hacia el conocimiento.

El estado del cómo utilizan las tecnologías digitales los investigadores en formación muestra una comunidad que, ante el desarrollo dinámico de las TIC, tiene una disparidad entre lo que sabe en términos disciplinares y la apropiación de su entorno educativo digital. Los grupos de investigación tienen hoy una capacidad única de comunicación, que se incrementa en sus formas y alcances, pero no han integrado los saberes necesarios para potenciar el aprendizaje en red.

Finalmente, es pertinente señalar que esta investigación identifica una dispersión de problemas referentes al uso de las tecnologías digitales por parte de los investigadores en formación, es decir, no hay una unificación de términos de búsqueda acerca del fenómeno. Esta situación se manifestó durante la etapa de determinación de criterios de búsqueda, pues los resultados arrojaron gran cantidad de estudios, al utilizar conceptos como "tecnología digital" o "problemas de aprendizaje". Ante este escenario, es conveniente continuar la búsqueda y selección de términos referentes a la temática abordada, paralelamente con la delimitación de los contextos relacionados 
con la región de estudio.

\section{REFERENCIAS}

Alexander, B., Ashford-Rowe, K., Barajas-Murphy, N., Dobbin, G., Knott, J., Mccormack, M., y Weber, N. (2019). Horizon Report. Higher Education Edition. Descargado de https://ibrary.educause.edu/-/media/files/library/2019/4/2019horizonreport.pdf?la= en\&hash=C8E8D444AF372E705FA1BF9D4FF0DD4CC6F0FDD1

Arras-Vota, A. M., Gutiérrez-Díez, M. C., y Bordas-Beltrán, J. L. (2010). Escenarios de aprendizaje y satisfacción estudiantil en posgrado virtual. Apertura, 9(1), 110-125. https://dx.doi.org/10.18381/Ap.v9n1.918

Becerra-Romero, A. T. (2017). Alcances y limitaciones del posgrado en la Universidad Autónoma de Nayarit. Revista de la Educación Superior. RESU, 46(183), 105-121. https://dx.doi.org/10.1016/j.resu.2017.05.003

Cabero-Almenara, J. (2013). Formación del profesorado universitario en TIC. Aplicación del método Delphi para la selección de los contenidos formativos. Educación XX, 1(17), 111-131. https://doi.org/10.5944/educxx1.17.1.10707

Carrasco, S., Baldivieso, S., y Di-Lorenzo, L. (2016). Una experiencia innovadora de enseñanza en el nivel superior en el contexto latinoamericano. RED. Revista de Educación a Distancia, 48(6), 1-19. https://doi.org/10.6018/red/48/6

Casimiro, W., Casimiro, C., y Casimiro, J. (2017). Apropiación de dinámicas de investigación en docentes universitarios. Un estudio fenomenológico. Opción, 33(83), 336-372. Descargado de https:/produccioncientificaluz.org/index.php/opcion/article/view/23119/23160

Chávez-Caraza, K. L., de Ita, J. R., Lozano-Ramírez, J. F., Vargas-Duarte, G. M., y Lozano-Lee, F. G. (2015). Desarrollo e implementación de un curso de investigación para estudiantes de ciencias de la salud: una propuesta para estimular la producción científica. Investigación en Educación Médica, 4(15), 161-169. https://dx.doi.org/10.1016/j.riem.2015.04.001

Conacyt. (2018). Manual del Sistema de Clasificación de Revistas Mexicanas de Ciencia y Tecnología. Ciudad de México: CONACyT. Descargado de http://www.revistascytconacyt.mx/manual-sistema-crmcyt.pdf

Conacyt. (2020). onsejo Nacional de Ciencia y Tecnología. Descargado de https://www.conacyt.gob.mx

Degkwitz, A. (2017). The Interactive Library as a Virtual Working Space. LIBER Quarterly. The Journal of the Association of European Research Libraries, 27(1), 127-137. https://dx.doi.org/10.18352/lq.10214

DOF. (2018). Ley General de Educación. Descargado de https://www.sep.gob.mx/work/models/ sep1/Resource/558c2c24-0b12-4676-ad90-8ab78086b184/ley_general_educacion.pdf

Fernández-Altunaa, M. A., del Prado, A. M., Arriarán-Rodríguez, E., Gutiérrez-Rayón, D., Toriz-Castillo, H. A., y Lifshitz-Guinzberg, A. (2016). Uso de los MeSH: una guía práctica. Investigación en Educación Médica, 5(20), 220-229.

https://dx.doi.org/10.1016/j.riem.2016.02.004

Ford, D. R. (2019). Pedagogy of the "Not": Negation, Exodus, and Postdigital Temporal Regimes. Postdigital Science and Education(1), 104-118. https://doi.org/10.1007/s42438-018-0009-4

Gisbert, J. P., y Bonfill, X. (2004). ¿Cómo realizar, evaluar y utilizar revisiones sistemáticas y metaanálisis? Gastroenterología y Hepatología, 27, 129-149. https://dx.doi.org/10.1016/s0210-5705(03)79110-9

Gómez-Rivas, J., Carrión, D. M., Tortolero, L., Veneziano, D., Esperto, F., Greco, F., ... Rodríguez-Socarrás, M. (2019). Las redes sociales científicas, una nueva forma de ampliar el conocimiento. ¿Qué necesitan saber los urólogos? Actas Urológicas 
Españolas, 43(5), 269-276. https://doi.org/10.1016/J.ACURO.2018.12.003

Gros, B. (2016). Retos y tendencias sobre el futuro de la investigación acerca del aprendizaje con tecnologías digitales. RED. Revista de Educación a Distancia(32), 1-13. Descargado de https://www.um.es/ead/red/32/gros.pdf

Gros, B., y Noguera-Fructuoso, I. (2013). Mirando el futuro: Evolución de las tendencias tecnopedagógicas en educación superior. Campus Virtuales, 2(2), 130-140. Descargado de http://uajournals.com/ojs/index.php/campusvirtuales/article/view/44/43

Guerrero-Martínez, J. A., y Reyes, J. C. (2018). High expression of SMARCA4 or SMARCA2 is frequently associated with an opposite prognosis in cancer. Scientific Reports, 8(2043). https://dx.doi.org/10.1038/s41598-018-20217-3

Hernández, L., Rodríguez, N., Andrés-Pérez, E., y Augusto-Reyes, D. (2017). Atlas interactivo de neuroimágenes y correlación clínico-imagenológica en ataque cerebrovascular agudo: recurso digital educativo. Repertorio de Medicina y Cirugía, 26(1), 50-53. https://dx.doi.org/10.1016/j.reper.2016.12.002

Jandrić, P. (2019). Editorial: Welcome to Postdigital Science and Education! Postdigital Science and Education(1), 1-3. https://doi.org/10.1007/s42438-018-0013-8

Jandrić, P., Knox, J., Besley, T., Ryberg, T., Suoranta, J., y Hayes, S. (2018). Postdigital science and education. Educational Philosophy and Theory, 50(10), 893-899.

https://dx.doi.org/10.1080/00131857.2018.1454000

López-Ledesma, A.-E. G., Álvarez, G., y Bassa, L. (2018). Educación virtual en la universidad: un estudio de Investigación-acción para la enseñanza de la asignatura Tecnología educativa. Ciencia, Docencia y Tecnología, 29(57), 201-234. Descargado de http://pcient.uner.edu.ar/cdyt/article/view/357

Marzal, M. A., Calzada-Prado, J., y Ruvalcaba-Burgoa, E. (2015). Objetos de aprendizaje como recursos educativos en programas de alfabetización en información para una educación superior de posgrado competencial. Investigación Bibliotecológica: Archivonomía, Bibliotecología e Información, 29(66), 139-168.

https://dx.doi.org/10.1016/j.ibbai.2016.02.029

Muirragui-Irrazábal, V. (2019). Soporte a la docencia por bibliotecas adscritas a la red de bibliotecas Universitarias Españolas. Revista Electrónica en Educación y Pedagogía, 3(4), 107-121. https://doi.org/10.15658/rev.electron.educ.pedagog19.03030407

Navarrete, L., y Pérez, C. (2019). Revistas biomédicas: desarrollo y evolución . Revista Médica Clínica Las Condes, 30(3), 219-225. https://dx.doi.org/10.1016/j.rmclc.2019.04.002

Negroponte, N. (1995). Being digital. New York: Alfred A. Knopf.

Negroponte, N. (1998, 12 de 01). Negroponte. Descargado de https://www.wired.com/1998/12/negroponte-55/

Núñez-Reiz, A., de-la Hoz, M. A. A., y Sánchez-García, M. (2019). Big Data Analysis y Machine Learning en medicina intensiva. Medicina Intensiva, 43(7), 416-426. https://dx.doi.org/10.1016/j.medin.2018.10.007

Olivares-Carmona, K. M., Angulo-Armenta, J., Torres-Gastelú, C. A., y Madrid-García, E. M. (2016). Las TIC en educación: metaanálisis sobre investigación y líneas emergentes en México. Apertura. Revista de Innovación Educativa, 8(2), 100-115.

http://dx.doi.org/10.18381/Ap.v8n2.866

Pepperell, R., y Punt, M. (2000). The Postdigital Membrane: Imagination, Technology and Desire. Bristol: Intellect.

Peters, M. A., y Jandrić, P. (2019). Posthumanism, open ontologies and bio-digital becoming: Response to Luciano Floridi's Onlife Manifesto. Educational Philosophy and Theory, 51(10), 971-980. https://doi.org/10.1080/00131857.2018.1551835

Pisté-Beltrán, S., y and, M.-A. M.-G.-Q. (2019). Bibliotecas universitarias y educación digital abierta: un espacio para el desarrollo de instrumentos de implementación en web, de competencias en información e indicadores para su evaluación. Revista Interamericana de Bibliotecología, 42(1), 277-288. https://dx.doi.org/10.17533/udea.rib.v41n3a06

Powell, W. W., y Snellman, K. (2004). The Knowledge Economy. Annual Review of Sociology, 
30(1), 199-220. https://doi.org/10.1146/annurev.soc.29.010202.100037

Rodríguez-Bravo, B., Alvite-Díez, M. L., y Olea-Merino, I. (2015). La utilización de las revistas electrónicas en la Universidad de León (España): hábitos de consumo y satisfacción de los investigadores. Investigación Bibliotecológica: Archivonomía, Bibliotecología e Información, 29(66), 17-55. https://dx.doi.org/10.1016/j.ibbai.2016.02.024

Rubio-Hurtado, M. J., Vilà-Baños, R., y Berlanga-Silvente, V. (2015). La Investigación Formativa Como Metodología de Aprendizaje en la Mejora de Competencias Transversales. Procedia - Social and Behavioral Sciences, 196(8), 177-182. https://dx.doi.org/10.1016/j.sbspro.2015.07.037

Salinas-Ibáñez, J., y Irene-Marin, V. (2018). Las diferentes concepciones de la universidad digital en Iberoamérica. RIED. Revista Iberoamericana de Educación a Distancia, 21, 97-97. https://dx.doi.org/10.5944/ried.21.2.20653

Sánchez-Meca, J. (2010). Como realizar una revision sistematica y un meta-analisis. Aula Abierta, 38(2), 53-64. Descargado de https://www.um.es/metaanalysis/pdf/5030.pdf

Sancho-Gil, J. M., Ornellas, A., y Arrazola-Carballo, J. (2018). La situación cambiante de la universidad en la era digital. RIED. Revista Iberoamericana de Educación a Distancia, 21(2), 31-49. https://dx.doi.org/10.5944/ried.21.2.20673

Santana, S. (2018). Las métricas alternativas y sus potencialidades para el profesional de la salud. Revista Médica Clínica Las Condes, 29(4), 484-490.

https://doi.org/10.1016/j.rmclc.2017.08.012

Sime-Poma, L. (2017). Grupos de investigación en educación: hacia una tipología multirreferencial desde casos representativos. Revista de la Educación Superior. RESU, 46(184), 97-116. https://dx.doi.org/10.1016/j.resu.2017.12.002

Vaccari, A. (2008). Reseña de "Reensamblar lo social: una introducción a la teoría del actor-red” de Bruno Latour. Revista iberoamericana de Ciencia, Tecnología y Sociedad CTS, 4(11), 189-192. Descargado de https://www.redalyc.org/articulo.oa?id=92441112 